\title{
Mistura PAni.DBSA/SBS Obtida por Polimerização "In Situ": Propriedades Elétrica, Dielétrica e Dinâmico-Mecânica
}

\author{
María E. Leyva, Guilherme 0. Barra, Bluma G. Soares \\ Instituto de Macromoléculas Professora Eloisa Mano, UFRJ \\ Dipak Khastgir \\ Rubber Technology Centre, Indian Institute of Technology, India
}

\begin{abstract}
Resumo: Misturas elastoméricas condutoras de eletricidade envolvendo copolímero tribloco poli(estireno-b-butadienob-estireno) (SBS) e polianilina dopada com ácido dodecilbenzenosulfônico (Pani.DBSA) foram obtidas por polimerização "in situ". Os filmes obtidos por moldagem por compressão mostraram baixo limiar de percolação, apresentando valores de condutividade semelhantes aos encontrados para o polímero condutor puro com cerca de 20 \% em massa de Pani.DBSA. A caracterização das misturas por análise termodinâmico-mecânica (DMTA) evidenciou uma ligeira interação da Pani.DBSA com ambas fases do copolímero SBS. Na região borrachosa, o módulo da mistura aumenta com o aumento do conteúdo de Pani. No entanto, existe uma progressiva queda no fator de amortecimento ("damping") com o aumento da concentração de Pani. A energia de ativação, Ea, do processo de transição vítreo-borrachoso de ambas fases do SBS foi calculada, utilizando a equação de Arrhenius com os dados obtidos tanto por DMTA como por análise termodielétrica (DETA). A caracterização dielétrica não proporcionou informações a respeito da localização da Pani.DBSA na matriz de SBS. No entanto, observou-se o fenômeno de polarização interfacial entre a Pani e o SBS. Uma morfologia do tipo microtubos foi observada para Pani.DBSA na mistura SBS/Pani.DBSA, utilizando-se a técnica de microscopia eletrônica de varredura.
\end{abstract}

Palavras-chaves: Polianilina, ácido dodecilbenzenossulfônico, SBS, polimerização "in situ”.

\section{PAni.DBSA/SBS blends prepared from "in situ” polymerization: electric, dielectric and dynamic-mechanical properties}

\begin{abstract}
Conducting rubbery blends of styrene-butadiene-styrene (SBS) triblock copolymer and polyaniline doped with dodecylbenzenesulfonic acid (Pani.DBSA) were produced by "in situ" polymerization. The films obtained by compression-molding display low percolation threshold with conductivity values similar to that found for pure Pani.DBSA with only $20 \mathrm{wt} \%$ of Pani.DBSA in the SBS/Pani.DBSA blend. The dynamic-mechanical characterization demonstrated that PAni.DBSA presents a slight interaction with both phases of the SBS copolymer. In the rubbery region, the modulus of the blend increases with the increase of the Pani content. The values of Tg of the polybutadiene block were not affected by the addition of Pani. However, there is a progressive drop of the damping values with the increase of Pani concentration. The activation energy Ea for the glass transition process for both SBS phases was calculated from DMTA and DETA results, using the Arrhenius equation. From DMTA data, it is found that Ea corresponding to the PB segment of SBS presents a slight increase with the addition of Pani.DBSA, whereas the Ea corresponding to the PS segment increases substantially with the presence of Pani.DBSA. These results were also confirmed by DETA. The dielectric characterization does not provide conclusive information concerning the preferential localization of Pani.DBSA, but gives some information about interfacial polarization between the Pani and SBS in this blend. The formation of microtubules was observed by scanning electron microscopy in the SBS/Pani.DBSA blends.
\end{abstract}

Keywords: Polyaniline, dodecylbenzenesulfonic acid, SBS, “in situ” polymerization.

Autor para correspondência: Bluma G. Soares, I MA/UFRJ, Caixa Postal: 68525, CEP : 21945-970, Rio de Janeiro, RJ. E-mail: bluma@ima.ufrj.br 


\section{Introdução}

Dentre os procedimentos relatados na literatura para obtenção de misturas entre polímeros condutores de eletricidade e polímeros isolantes, consideramos de grande interesse a mistura por polimerização "in situ", uma vez que este procedimento permite obter em uma única etapa, um material polimérico facilmente processável e com relativamente alta condutividade elétrica.

O emprego de copolímeros em bloco poli(estireno-b-butadieno-b-estireno), SBS, como matriz isolante em misturas com polímeros condutores é de grande importância devido à sua dupla natureza de elastômero e termoplástico, o que permite um fácil processamento e boas propriedades mecânicas sem a necessidade de vulcanização ${ }^{[1]}$. Alguns artigos na literatura cientifica empregam este copolímero como matriz em misturas condutoras, obtidas a partir da polimerização química "in situ" da anilina ${ }^{[2]}$ ou pirrol $^{[3]}$. Misturas de SBS com polianilina (Pani) dopada com ácido dodecil benzeno sulfônico (DBSA), preparadas no estado fundido ou em solução, têm sido estudadas por nosso grupo de pesqui$\mathrm{sa}^{[4,5]}$. Na primeira técnica ${ }^{[4]}$, o complexo Pani.DBSA foi obtido por redopagem da base de polianilina com DBSA a partir da mistura mecânica de ambos os componentes em gral. Na segunda técnica ${ }^{[5]}$, conduzida em solução, o complexo Pani.DBSA foi obtido através do método conhecido como "polimerização com dopagem in situ" onde a anilina é polimerizada em emulsão na presença do dopante DBSA, que atua simultaneamente como estabilizante da emulsão e dopante da Pani.

Segundo a literatura ${ }^{[6]}$, a morfologia do SBS exibe duas fases bem definidas e segregadas, devido à incompatibilidade termodinâmica entre os blocos de poliestireno (PS) e polibutadieno (PB). Se a polianilina dopada com ácido dodecil benzeno sulfônico (Pani.DBSA) fosse compatível só com uma das duas fases do SBS, o limiar de percolação da mistura poderia ser controlado, utilizando-se copolímeros com diferentes teores de PS e PB.

A introdução da PAni.DBSA na matriz do SBS modifica as relaxações dielétricas e dinâmico-mecânicas do SBS, além das propriedades elétricas e mecânicas. O estudo dessas relaxações poderia fornecer importantes indicações sobre a fase do SBS onde se localiza preferencialmente a Pani.DBSA.
Este trabalho tem como objetivo a preparação de misturas condutoras de eletricidade envolvendo Pani.DBSA/SBS, em uma única etapa, através da polimerização da anilina em emulsão de água em um meio orgânico onde o SBS esteja dissolvido. O DBSA estabilizaria a emulsão e ao mesmo tempo atuaria como dopante da Pani. Além das propriedades elétricas das misturas, estudaremos suas características dielétricas, dinâmico-mecânicas e a morfologia da Pani.DBSA na matriz de SBS. Além disso, pretendese obter informações sobre a localização do polímero condutor na matriz isolante, a partir dos resultados das relaxações dielétricas e dinâmico-mecânicas da mistura PAni.DBSA/SBS.

\section{Experimental}

\section{Materiais}

A anilina (Merck) foi destilada sob vácuo e armazenada em geladeira. O persulfato de amônio $\left(\mathrm{NH}_{4}\right)_{2} \mathrm{~S}_{2} \mathrm{O}_{8}$ (Merck, grau analítico) e o ácido dodecil benzeno sulfônico, DBSA (Pro-Química do Brasil, grau técnico), foram utilizados sem prévia purificação. O copolímero em bloco poli(estireno-cobutadieno-co-estireno) SBS (TR-1061) (conteúdo de $\mathrm{PB}=70 \%$ em peso; $\left.\mathrm{M}_{\mathrm{w}}=120,000\right)$ foi gentilmente cedido pela Petroflex S.A. (Rio de Janeiro, Brasil).

\section{Obtenção da mistura Pani.DBSA/SBS}

O SBS e a anilina foram dissolvidos em tolueno sob agitação mecânica. Em seguida, uma solução de DBSA em tolueno foi lentamente adicionada. A emulsão foi formada sob vigorosa agitação quando se adicionou água destilada. Finalmente adicionouse, gota a gota, a solução aquosa de oxidante, $\left(\mathrm{NH}_{4}\right)_{2} \mathrm{~S}_{2} \mathrm{O}_{8}$. A polimerização foi mantida entre $0-5^{\circ} \mathrm{C}$ durante $6 \mathrm{~h}$. Finalizado o tempo, a emulsão foi desestabilizada precipitando-se a mistura em metanol. O precipitado foi lavado com metanol e seco a vácuo. As proporções dos componentes da reação foram: $\left(\mathrm{NH}_{4}\right)_{2} \mathrm{~S}_{2} \mathrm{O}_{8} / \mathrm{An}=0,4$ molar; DBSA/Anilina $=$ 1,5 molar; tolueno/água $=8 / 2(\mathrm{v} / \mathrm{v}) ;$ SBS/Anilina $=0,1$; 0,$15 ; 0,2 ; 0,3 ; 1,0 ; 2,0 ; 3,0$ (razão em massa).

\section{Caracterização}

A concentração de Pani na mistura foi determinada por análise elementar no equipamento PerkinElmer 2400. As misturas Pani.DBSA/SBS foram moldadas por compressão a $130{ }^{\circ} \mathrm{C}$ durante $7 \mathrm{~min}$, a 
uma pressão de 1,0 MPa, em placas de $16 \mathrm{~cm}^{2}$ e cerca de $1 \mathrm{~mm}$ de espessura. As medidas de condutividade foram feitas através do método padrão de quatro pontas utilizando um eletrômetro Keithley 617 e fonte de corrente Keithley 224.

O estudo da relaxação dielétrica foi realizado em analisador térmico dielétrico DETA (TA Instruments, modelo 2970) empregando eletrodos de placas paralelas. O DETA foi utilizado para determinar a constante $\varepsilon$ ' e a perda dielétrica $\varepsilon$ " na faixa de temperatura - 130 a $180{ }^{\circ} \mathrm{C}$ e de freqüência de 0,3 a $10^{5} \mathrm{~Hz}$, sob atmosfera de nitrogênio. Para estudar as propriedades dielétricas, a mistura condutora foi submetida a um processo de desdopagem com solução aquosa de $\mathrm{NH}_{4} \mathrm{OH} 0,1 \mathrm{M}$ durante 24 horas.

O estudo das relaxações dinâmico-mecânicas foi realizado em um analisador térmico dinâmico mecânico DMTA (Rheometric Scientific, modelo MK III). O DMTA foi utilizado para determinar o modulo de flexão E' e a tangente da perda mecânica Tanઈ, na faixa de temperatura -130 a $130{ }^{\circ} \mathrm{C}$, à freqüência de $1 \mathrm{~Hz}$. A energia de ativação correspondente às transições vítreo-borrachosa das duas fases do SBS foi determinada por DMTA, a partir de determinações realizadas a freqüências de 1,10 e $100 \mathrm{~Hz}$.

A morfologia da mistura PAni.DBSA/SBS foi estudada a partir de análises de microscopia eletrônica de varredura (SEM), em um microscópio Jeol JSM-5300 operando a $20 \mathrm{kV}$. O material prensado foi fraturado criogenicamente, sendo analisada a superfície de fratura coberta por uma fina camada de ouro.

\section{Resultados e Discussão}

\section{Propriedades elétricas}

A Tabela 1 apresenta a composição de cada uma das misturas obtidas através da técnica de polimerização "in situ" em emulsão, bem como os respectivos valores de condutividade elétrica. A concentração de Pani foi determinada pela técnica de análise elementar. Para expressar os valores obtidos por $\mathrm{CHN}$ em termos de fração volumétrica, determinou-se a densidade do SBS empregado na mistura $\left(0,89 \mathrm{~g} / \mathrm{cm}^{3}\right)$ e da Pani obtida através de síntese em emulsão $\left(1,15 \mathrm{~g} / \mathrm{cm}^{3}\right)$.

A mudança da condutividade elétrica nas misturas Pani.DBSA/SBS com o aumento da concentração de Pani é mostrada na Figura 1.

Do comportamento elétrico das misturas Pani.DBSA/SBS observado na Figura 1 podemos con-
Tabela 1. Composição e condutividade elétrica das misturas Pani.DBSA/ SBS preparadas "in situ"

\begin{tabular}{ccccc}
\hline An:SBS & $\begin{array}{c}\text { SBS } \\
(\mathbf{g})\end{array}$ & $\begin{array}{c}\text { Pani } \\
(\%)\end{array}$ & $\begin{array}{c}\text { Pani } \\
\text { (fração vol) }\end{array}$ & $\begin{array}{c}\boldsymbol{\sigma} \\
\left(\mathbf{S c m}^{-1}\right)\end{array}$ \\
\hline $0: 100$ & 100,0 & 0,00 & 0,000 & $7 * 10^{-16}$ \\
$1: 9,99$ & 24,0 & 3,48 & 0,046 & $2 * 10^{-7}$ \\
$1: 6,66$ & 16,0 & 6,97 & 0,092 & $9 * 10^{-6}$ \\
$1: 4,99$ & 12,0 & 2,33 & 0,031 & $1 * 10^{-7}$ \\
$1: 3,33$ & 8,0 & 13,95 & 0,179 & 0,02 \\
$1: 1,00$ & 2,4 & 29,07 & 0,356 & 0,28 \\
$1: 0,49$ & 1,2 & 34,88 & 0,419 & 0,07 \\
$1: 0,42$ & 0,8 & 55,81 & 0,630 & 0,15 \\
$1: 0,00$ & 0,0 & 100,00 & 1,000 & 0,70 \\
\hline
\end{tabular}

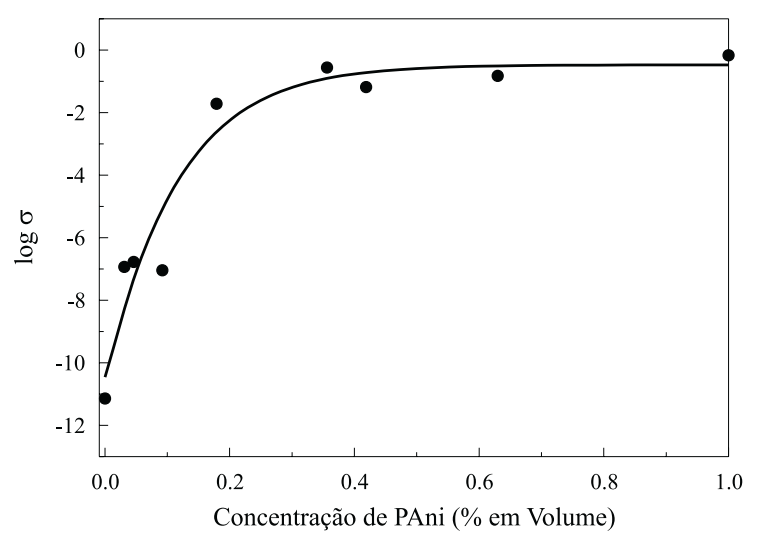

Figura 1. Comportamento elétrico das misturas Pani.DBSA/SBS em função da concentração de Pani.DBSA

cluir que o limiar de percolação se situa em torno de $0,1 \%$ em volume de Pani (cerca de $7 \%$ em peso). Este baixo valor do limiar de percolação é reportado na literatura para misturas de Pani.DBSA com outros polímeros, obtidas por esta técnica ${ }^{[7,8]}$. O limiar de percolação encontrado para estas misturas é bem baixo quando comparado com aqueles encontrados para compósitos poliméricos condutores a base de Negro de Fumo ( $20 \%$ em peso de Negro de Fumo). Este comportamento é explicado pela diferente morfologia apresentada por estes dois materiais condutores $^{[7]}$. O negro de fumo apresenta uma estrutura de agregados, enquanto que a Pani quando dopada com DBSA ou outros dopantes orgânicos forma uma estrutura tubular interconectada, a qual permite a formação da rede condutora a baixas concentrações do polímero condutor. A condutividade máxima atingida para as misturas foi de $10^{-1} \mathrm{Scm}^{-1}$. É importante 
destacar que este valor é próximo àquele obtido para a Pani.DBSA pura e que a mistura com $20 \%$ em peso de Pani já atinge o máximo valor de condutividade.

\section{Propriedades dinâmico-mecânica}

O comportamento dinâmico-mecânico de misturas SBS/Pani.DBSA foi comparado com aquele do SBS puro. As misturas foram preparadas adicionandose 24 e 8 g de SBS respectivamente na polimerização em emulsão da anilina sob idênticas condições de agitação mecânica, tempo e temperatura de reação para evitar o efeito das condições de reação nas propriedades finais das misturas. As misturas continham, portanto, 3,45 \% e 13,95\% de Pani.DBSA, respectivamente. A Figura 2 ilustra a variação do modulo de flexão com a temperatura na faixa de -120 a $120^{\circ} \mathrm{C}$. O SBS puro apresenta uma súbita queda no módulo ao redor de $-80^{\circ} \mathrm{C}$, o que corresponde à transição vítrea - borrachosa do bloco de polibutadieno no copolímero SBS.

Quando o SBS se mistura com o complexo Pani.DBSA através da polimerização "in situ", observa-se que a temperatura de transição vítrea do bloco de polibutadieno permanece inalterada. No entanto, o módulo elástico na região borrachosa (entre $-80^{\circ} \mathrm{C}$ a $90{ }^{\circ} \mathrm{C}$ ) sofre um significativo acréscimo com o aumento da concentração de Pani na mistura. Este aumento do módulo pode ser atribuído ao aumento da rigidez do sistema devido à incorporação de Pani. Informação adicional pode ser obtida a partir do gráfico da tangente de perda mecânica vs. temperatura, apresentado na Figura 3.

No gráfico da Tan $\delta$ vs. temperatura observa-se um pico agudo a $-81{ }^{\circ} \mathrm{C}$ na curva correspondente ao SBS puro, sendo que este pico permanence inalterá-



Figura 2. Dependência do Log E' com a temperatura para o SBS puro e sua mistura com Pani.DBSA.

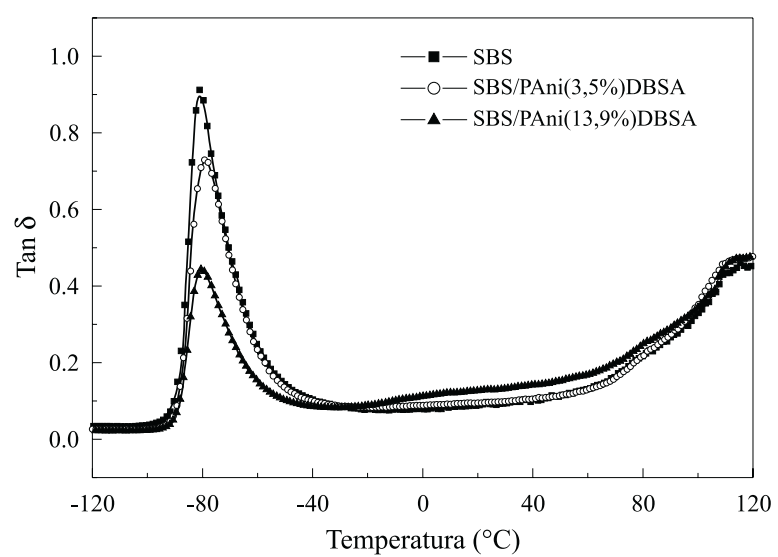

Figura 3. Comportamento da Tan $\delta$ com a temperatura para o SBS puro e sua mistura com Pani.DBSA.

vel quando se adiciona 3,48 e $13,95 \%$ em peso de Pani. No entanto observa-se um progressivo decréscimo na altura do pico com o aumento da concentração de Pani. Este decréscimo nos valores de Tan $\delta$ representa o decréscimo do "damping" do sistema ${ }^{[9]}$ ocasionado provavelmente pela influência da Pani rígida no segmento flexível de polibutadieno. Isto pode ser explicado pela Tg da Pani pura que possui, de acordo com a literatura, um valor muito mais elevado $^{[10]}$. Uma situação semelhante é encontrada quando partículas rígidas são adicionadas a matrizes elastoméricas flexíveis ${ }^{[9]}$.

Geralmente, a adição de fibras rígidas a matrizes poliméricas flexíveis ocasiona um aumento da rigidez, o que conduz a um deslocamento dos valores de Tg para temperaturas mais altas. No entanto, isto não foi observado em nosso sistema. Acredita-se que, neste caso, as moléculas de DBSA estejam também atuando como plastificante. Segundo a literatura ${ }^{[9]}$ a adição de pequenas quantidades de plastificante a um polímero pode provocar uma redução significativa da temperatura de transição vítrea. Em nosso sistema, entretanto, a cadeia principal de Pani age como uma fibra rígida e o contra-íon, DBSA, como plastificante. Como a concentração de DBSA é pequena o efeito da Pani é mais pronunciado, provocando a redução do damping sem deslocar os valores de Tg.

Com relação ao segmento de PS, a adição de Pani não afetou os valores de $\tan \delta$

Seria interessante conhecer a interação do complexo Pani.DBSA com estas duas fases do copolímero em bloco SBS. Para obter informação sobre esta interação resolvemos determinar a energia de ativação Ea do processo de transição vítreo - borrachosa para ambas as fases, a partir dos picos corresponden- 
Tabela 2. Valores de Ea da transição vítrea-borrachosa das fases PB e PS obtidos por DMTA.

Energia de ativação Ea (Kcalmol-1)

\begin{tabular}{lcc}
\cline { 2 - 3 } & SBS & SBS/Pani(3,48\%)DBSA \\
Bloco de PB & 30 & 32 \\
Bloco de PS & 127 & 134 \\
\hline
\end{tabular}

tes à Tg obtidos a diferentes freqüências e utilizando a equação de Arrenhius, a qual é aplicável com alguma aproximação para estas transições ${ }^{[1]}$ :

$$
f=f_{0} e^{(E a / R T)}
$$

onde $f_{0}$ corresponde à freqüência a temperatura infinita.

A partir do coeficiente angular da reta que melhor ajusta os pontos experimentais no gráfico do $\log f$ $(\mathrm{Hz})$ vs. $1 / \mathrm{T}\left(\mathrm{K}^{-1}\right)$, obtem-se os valores de energia de ativação apresentados na Tabela 2.

Dos cálculos realizados encontramos que a Ea do bloco de PB é similar em ambos os sistemas SBS puro e SBS/Pani.DBSA. O cálculo da Ea da transição vítrea para a fase $\mathrm{PB}$ é realizado com maior exatidão através da equação Williams-Landel-Ferry $(\mathrm{WLF})^{[12]}$. O valor reportado na literatura e calculado pela equação WLF corresponde ao valor obtido neste trabalho ${ }^{[11]}$.

No caso da fase PS, encontramos um ligeiro aumento da Ea quando o complexo Pani.DBSA é adicionado ao SBS (Tabela 2). Este aumento da Ea do processo de transição vítrea - borrachosa do bloco PS na mistura Pani.DBSA/SBS comparado ao SBS puro, significa que existe uma maior interação do complexo Pani.DBSA com o bloco de PS.

\section{Propriedades dielétricas}

A Figura 4 mostra a relaxação dielétrica do SBS puro representada em termos da perda dielétrica vs. temperatura a diferentes freqüências. O SBS puro exibe três relaxações dielétricas diferentes: a primei$\mathrm{ra}$, entre -80 e $-70^{\circ} \mathrm{C}$, corresponde à transição vítrea do bloco de PB; a segunda, entre 90 e $120^{\circ} \mathrm{C}$, que corresponde à transição vítrea do bloco de PS. Ambos os valores de Tg correspondem àqueles encontrados pela técnica de DMTA (Figura 3). Observe-se na Figura 4 que o pico relativo à Tg do PS só é detectado a freqüências superiores a $10 \mathrm{~Hz}$. Para freqüências inferiores a $10 \mathrm{~Hz}$, a perda dielétrica $\varepsilon$ " manifesta um agudo incremento com o incremento da tempera-

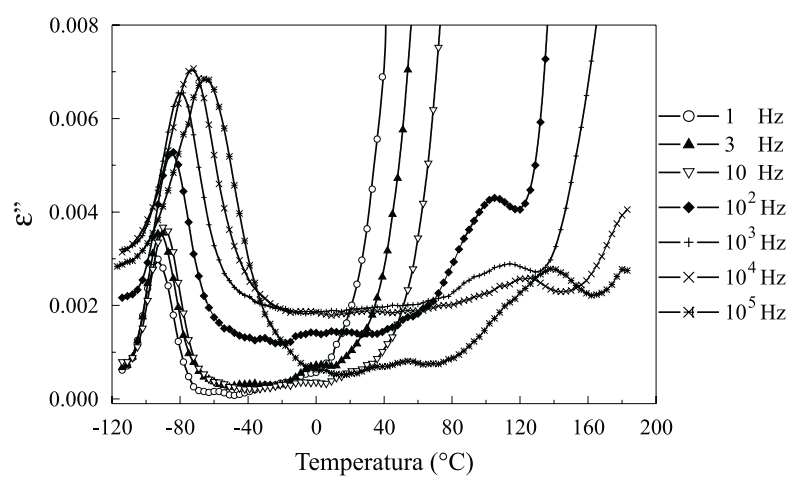

Figura 4. Comportamento de $\varepsilon$ " com a temperatura a diferentes freqüências para o SBS puro

tura o qual é conseqüência de uma forte contribuição da condutividade iônica à perda dielétrica. Ambos os picos, correspondentes aos blocos de PB e PS, deslocam-se para temperaturas mais elevadas com o aumento da frequência de 1 para $10^{5} \mathrm{~Hz}$. A terceira relaxação é observada entre 160 e $200{ }^{\circ} \mathrm{C}$ a freqüências de $10^{4}$ e $10^{5} \mathrm{~Hz}$. Segundo a literatura, esta transição pode aparecer em um copolímero em bloco e corresponde à transição de um sistema heterogêneo para um sistema homogêneo, onde os domínios desaparecem e os dois componentes (polibutadieno e poliestireno) misturam-se intimamente ${ }^{[11]}$. Esta temperatura onde acontece a transição de um "sistema estruturado para um sistema não estruturado" se conhece como temperatura de separação $T s^{[11]}$.

A dependência da perda dielétrica com a temperatura, correspondente à mistura Pani $(3,48 \%) / \mathrm{SBS}$, é mostrada na Figura 5. Para realizar este estudo dielétrico foi necessário diminuir a condutividade da mistura, desdopando-a com solução aquosa de $\mathrm{NH}_{4} \mathrm{OH} 0,1 \mathrm{M}$.

Como no caso do SBS puro, observa-se também, três relaxações dielétricas diferentes: a primeira ao redor de $-80^{\circ} \mathrm{C}$ relacionada à transição vítrea do bloco de PB. Este valor é semelhante ao encontrado por DMTA. Os picos correspondentes a esta transição são pequenos e largos e, portanto, foram ampliados para melhor observação. Os valores de perda dielétrica também são pequenos e isto se explica porque a resposta dielétrica da fase $\mathrm{PB}$ fica mascarada devido à elevada contribuição que a condutividade da Pani introduz na perda dielétrica da mistura. A segunda relaxação dielétrica é observada na faixa de -40 a $40^{\circ} \mathrm{C}$, a qual atribuímos à relaxação correspondente à polarização interfacial entre a matriz de SBS e a Pani, o qual é lógico que aconteça devido à pronunciada diferença entre os valores de condutividade elétrica de 


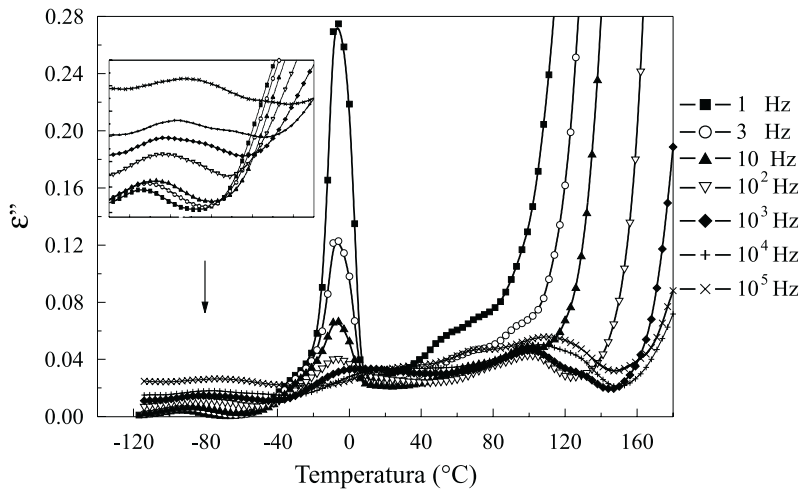

Figura 5. Comportamento de $\varepsilon$ " com a temperatura a diferentes freqüências para a mistura Pani(3,48\%)/SBS.

ambos os materiais. Para chegarmos a esta conclusão levamos em conta diferentes critérios que serão comentados a seguir. A terceira relaxação observada na Figura 5 encontra-se entre 90 e $120^{\circ} \mathrm{C}$ e corresponde à transição vítrea do bloco de PS, como encontrado também a partir de análise por DMTA.

Para atribuir a segunda relaxação à polarização interfacial entre a Pani e o SBS, realizamos primeiro o estudo dielétrico da Pani pura. A Pani pura obtida por polimerização em emulsão na presença de DBSA e posteriormente desdopada apresenta um comportamento dielétrico como mostrado na Figura 6.

Observa-se que, para medidas realizadas a valores pequenos da freqüência, a perda dielétrica mostra um rápido aumento com o aumento da temperatura. À medida que se aumenta a freqüência, a dependência de $\varepsilon$ " com a temperatura é mais suave, tornandose mais aguda após $-40^{\circ} \mathrm{C}$.

Segundo a teoria dielétrica, a perda dielétrica é proporcional à condutividade dos materiais ${ }^{[13]}$. $\mathrm{O}$ comportamento observado na Figura 6 demonstra que a Pani ainda no estado desdopado é um material com características semicondutoras, indicadas pelo aumento da condutividade com a temperatura. $\mathrm{Na}$ faixa de freqüência estudada, não se observa relaxação dielétrica alguma na Pani, observando-se unicamente as características semicondutoras do polímero.

Na Figura 5, o pico de $\varepsilon$ " correspondente a segunda relaxação apresenta um ligeiro deslocamento para maiores temperaturas com aumento da frequência o que possibilitou calcular a energia de ativação Ea do processo de relaxação a partir da equação de Arrenihus. O valor de Ea obtido foi de aproximadamente $20 \mathrm{Kcalmol}^{-1}$, o qual pode ser um critério a mais para afirmar que esta relaxação pode corresponder à polarização interfacial entre a Pani e o SBS,

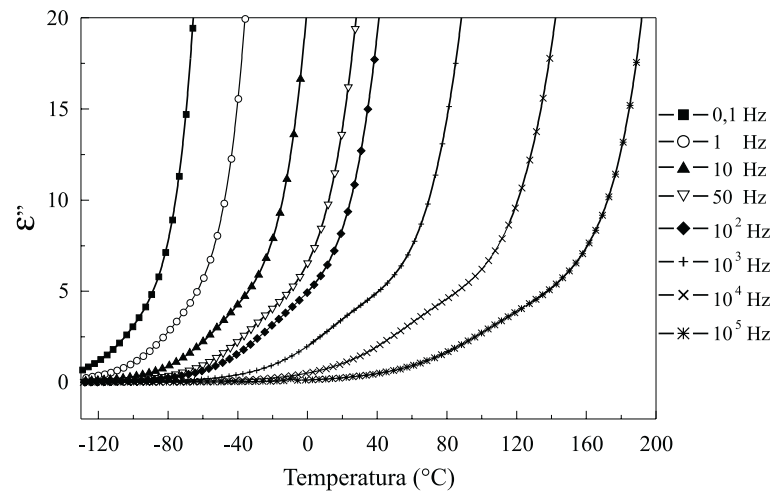

Figura 6. Dependência de $\varepsilon "$ com a temperatura a diferentes freqüências para a Pani pura

pois a Ea de relaxações correspondentes à polarização interfacial se caracterizam por valores baixos da energia de ativação.

O estudo dielétrico da mistura Pani(13,95\%) DBSA/SBS foi realizado na faixa de temperatura de -130 a $150{ }^{\circ} \mathrm{C}$. O comportamento dielétrico observado é muito parecido ao encontrado para a Pani pura, quer dizer, $\varepsilon$ " aumenta com a temperatura para toda a faixa de freqüências estudada, impedindo a obtenção de informação sobre as relaxações dielétricas das fases PB e PS do copolímero em bloco SBS. Consideramos que isto seja devido à maior condutividade da mistura, pois apesar da amostra ter sido neutralizada com solução aquosa de $\mathrm{NH}_{4} \mathrm{OH} 0,1 \mathrm{M}$, o maior teor de Pani na mistura faz com que prevaleçam as características semicondutoras deste polímero, mascarando qualquer outra informação correspondente às relaxações dielétricas correspondentes às fases $\mathrm{PB} e$ PS do copolímero SBS.

Como não foi possível obter informação sobre a relaxação dielétrica das fases PB e PS do copolímero SBS na mistura com 13,95 \% de Pani, decidimos realizar o estudo dielétrico para uma composição de Pani intermediária entre 3,48 e 13,95\%. A seguir são apresentados os resultados obtidos na caracterização dielétrica da mistura Pani(6,97\%).DBSA/SBS.

A Figura 7 mostra as características dielétricas da mistura Pani/SBS com 6,97 \% em peso de Pani. Esta mistura foi neutralizada sob as mesmas condições reportadas anteriormente. Pode-se observar que uma maior concentração de Pani causa uma maior perda dielétrica do sistema devido ao aumento da condutividade, o que impossibilita a observação da relaxação dielétrica correspondente à $\mathrm{Tg}$ da fase PS. No entanto, a baixas temperaturas, onde a característica semicondutora da Pani é menor, pode-se observar a 




Figura 7. Dependência de $\varepsilon$ " com a temperatura a diferentes freqüências para a mistura Pani(6,97 \%)DBSA/SBS

relaxação correspondente a Tg do bloco de PB. Com o aumento da temperatura e conseqüentemente da condutividade e da perda dielétrica, a observação da relaxação correspondente ao bloco de PS fica comprometida. No entanto, a altas temperaturas, na faixa correspondente à transição do "sistema estruturado a um sistema não estruturado" do copolímero em bloco SBS pode-se observar a relaxação dielétrica do SBS à temperatura que se conhece como temperatura de separação Ts. Observa-se que o pico correspondente à Ts não muda com a freqüência de medição. Este comportamento é diferente daquele observado para as relaxações correspondentes à $\mathrm{Tg}$, o que confirma que esta relaxação não corresponde à transição de segunda ordem.

Como observado na Figura 4 (SBS puro), na Figura 5 (Pani3,48\%/SBS) e na Figura 7 (Pani6,97\%/SBS), as posições dos picos de perda dielétrica atribuídos às transições vítreas das fases PB e PS do copolímero SBS encontram-se deslocadas para temperaturas mais altas com o aumento da freqüência utilizada na medida. Este comportamento permite determinar a energia de ativação relativa à transição vítrea das fases PS e PB através da equação de Arrenihus e poderá fornecer alguma informação a respeito das interações entre a Pani e os componentes do copolímero.

A Tabela 3 sumariza os valores de Ea dos blocos de PS e de PB encontrados para os dois sistemas: SBS puro e misturas envolvendo SBS/Pani.DBSA. Observa-se que a Ea do bloco de PB é menor no SBS puro do que nas misturas contendo Pani.DBSA. Comportamento semelhante foi também observado por DMTA, porém, esta diferença é mais significativa nos experimentos realizados por DETA (Tabela 3). Considerando a fase PS, o aumento da Ea é mais acentuado quando o complexo Pani.DBSA é adicionado ao SBS (Tabela 3).

A partir dos resultados obtidos por DETA, sugere-se que a presença de Pani.DBSA na matriz de SBS aumenta a Ea relacionada à transição vítrea de ambas as fases do copolímero em bloco.

As diferenças entre os valores de Ea obtidos por DETA e por DMTA são atribuídas à presença da Pani, uma vez que a sua característica condutora influencia significantemente as características dielétricas de ambas as fases do copolímero. Este fenômeno impossibilita a observação de qualquer interação especifica entre alguma das fases da matriz isolante e o polímero condutor.

Tabela 3. Valores de Ea relacionada à transição vítrea das fases PB e PS obtidas por DETA.

\begin{tabular}{cc}
\hline \multicolumn{2}{c}{$\begin{array}{c}\text { Energia de ativação Ea } \\
\text { (Kcalmol- }{ }^{-} \text {) }\end{array}$} \\
\hline Bloco de PB & Bloco de PS \\
31 & 134 \\
37 & 147 \\
36 & - \\
\hline
\end{tabular}






Pani(3,48\%)DBSA/SBS

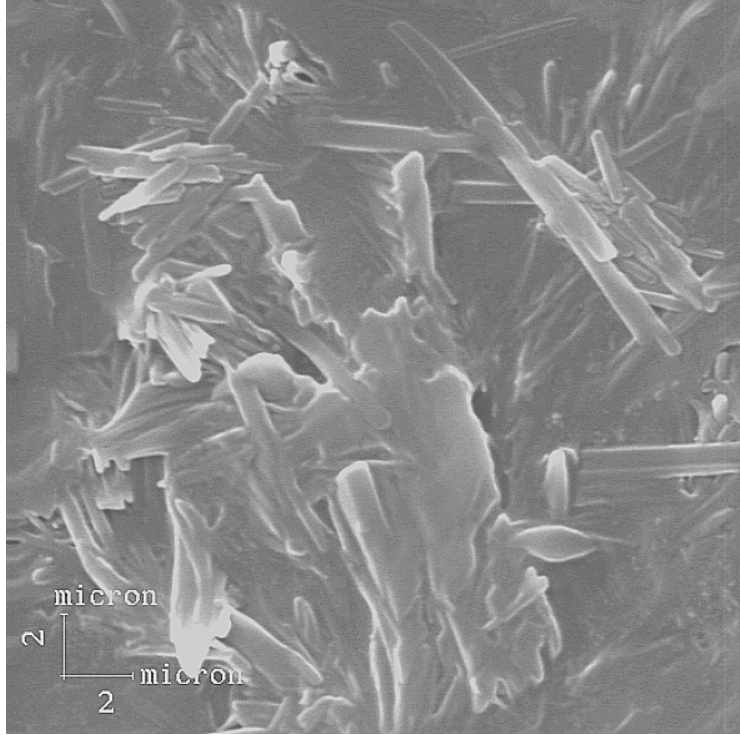

Pani(6,97\%)DBSA/SBS

Figura 8. Morfologia da superfície de fratura das misturas Pani.DBSA/SBS obtida por SEM.

\section{Microscopia eletrônica.}

As micrografias obtidas por SEM, das misturas Pani.DBSA/SBS com diferentes teores de Pani.DBSA são apresentadas na Figura 8. Observa-se que a Pani.DBSA apresenta uma morfologia tubular. A morfologia da mistura correspondente a composição anterior ao limiar de percolação, (Pani.DBSA $(3,48 \%) /$ SBS), apresenta agregados tubulares de Pani.DBSA isolados na matriz do SBS. No caso da mistura acima do limiar de percolação, a estrutura tubular da Pani.DBSA forma uma rede interconectada através da matriz isolante, permitindo assim a condução elétrica na mistura. Esta morfologia tubular justifica o baixo limiar de percolação obtido na mistura.

\section{Conclusões}

A polimerização "in situ" da anilina na presença de SBS e do dopante DBSA, permite obter uma mistura com baixo limiar de percolação. A mistura com $20 \%$ em peso de Pani atinge um valor de condutividade semelhante ao obtido para o polímero condutor puro dopado com DBSA e obtido pela mesma técnica de polimerização.

O complexo Pani.DBSA consta de duas partes: uma parte corresponde à Pani, a qual é rígida e age como fibra, e a outra parte corresponde ao DBSA, o qual age como plastificante. Devido a estas características do complexo, o aumento da concentração de Pani.DBSA na mistura origina uma progressiva queda nos valores de Tan $\delta$ sem ocasionar mudanças na Tg.

Dos valores da Ea relativa à transição vítrea das fases PB e PS do copolímero SBS obtidos a partir de DMTA, concluímos que existe uma maior interação do complexo Pani.DBSA com o bloco de PS se comparado ao bloco de PB. No entanto, foi observado também que a Pani afeta marcadamente o módulo de flexão na região borrachosa da fase PB. Portanto, a partir dos resultados obtidos por DMTA podemos concluir que o complexo influencia ambas as fases do copolímero em bloco SBS. Esta conclusão é razoável, pois a Pani têm uma estrutura quimicamente compatível com o bloco de PS, o que justificaria a interação física entre a Pani e o bloco PS. Porém, o contra-íon do DBSA presente nas cadeias de Pani, que é quimicamente compatível com o bloco de $\mathrm{PB}$, pode ser responsável pela interação da Pani com o bloco de PB.

Atribuímos a relaxação dielétrica observada na faixa de -40 a $40{ }^{\circ} \mathrm{C}$ na mistura Pani( $\left.3,48 \%\right) / \mathrm{SBS}$, à presença do fenômeno de polarização interfacial que pode ser estabelecido entre a Pani e o SBS, devido à enorme diferença entre os valores de condutividade de ambos os polímeros.

De acordo aos valores Ea da transição vítrea para a fase PB e PS do copolímero SBS obtidos a partir de DETA, concluímos que ambas as fases do copolímero PB e PS são afetadas pela presença da Pani e isto foi atribuído aos altos valores de condutividade deste polímero que influenciam as propriedades dielétricas 
das duas fases do SBS. Conclui-se, portanto que não é possível definir uma interação especifica entre a Pani e alguma das duas fases do SBS a partir dos resultados obtidos no DETA.

Com relação à localização do complexo em uma das duas fases do copolímero em bloco SBS podemos concluir, de acordo aos resultados obtidos por DMTA, que o complexo Pani.DBSA interage fisicamente com ambas fases do SBS.

\section{Agradecimentos}

Os autores agradecem à CAPES, CEPG/UFRJ, FAPERJ, CNPq e PADCT/CNPq (Proc. № 620132/ 98-1) e PADCT/FINEP (Proc. № 8898.0558.00) pelo apoio financeiro recebido.

\section{Referências Bibliográficas}

1. Nakajima, N. - Rubber Chem. Technol., 69, p.73 (1987).

2. Xie, H. Q.; Ma, Y. M. \& Guo, J. S. - Polymer, 40, p.261 (1998).

3. Ruckenstein, E. \& Hong, L. - Synth. Met., 66, p.249 (1994).
4. Leyva, M. E.; Barra, G. M. O.; Gorelova, M. M.; Soares, B. G. \& Sens, M. - J. Appl. Polym. Sci., 80, p.626 (2001).

5. Leyva, M. E.; Barra G. M. O. \& Soares, B. G. - Synth. Met., 123, p.443 (2001).

6. Van der Berg, P.; De Groot, H.; Van Dijk, M.A. \& Denley, D. R. - Polymer, 35, p.5778 (1994).

7. Oh, S. Y.; Koh, H. C.; Chol, J. W.; Rhee, H. W. \& Kim, H. S. - Polym. J., 29, p.404 (1997).

8. Xie, H. Q. \& Ma, Y. M. - J. Appl. Polym. Sci., 76, p.845 (1999).

9. Menard, K. P. - "Dynamic Mechanical Analysis: A Practical Introduction”, CRC Press, New York (1999).

10. Wei, Y.; Jang, G. W.; Hsueh, K. F.; Scherr, E. M.; MacDiarmid, A. G. \& Epstein, A. J. - Polymer, 33, p.314 (1992).

11. Shen, M. \& Kaniskin, V. A. - J. Polym. Sci.: Part B. Polym. Phys., 11, p.2261 (1973).

12. Tobolsky, A. V. - "Properties and Structure of Polymers", John-Wiley, New York (1960).

13. Pingsheng, H.; Xiaohua, Q. \& Chune, L. - Synth. Met., 55-57, p.5008 (1993).

Recebido: 10/08/01

Aprovado: 02/07/02 